\title{
coress feedback
}

Vascular surgeons, neurosurgeons and other surgical specialists often have to tunnel grafts, lines or drains subcutaneously. While this is a common and routine procedure in most cases, there is significant risk of entering a body cavity, hollow viscus or solid organ if the tunnelling device is misdirected inadvertently or if inappropriate force is employed. The series of cases reported here remind readers of those risks. Think before you tunnel!

We are grateful to the clinicians who have provided the material for these reports. The online reporting form is on our website (www.coress.org.uk), which also includes all previous Feedback Reports. Published contributions will be acknowledged by a 'Certificate of Contribution', which may be included in the contributor's record of continuing professional development.

Frank CT Smith Programme Director, on behalf of the CORESS Advisory Board

\section{Tunnel vision 1}

A 61-year-old man with extensive cardiovascular disease was admitted with sudden onset of bilateral leg ischaemia. Four years earlier, he had undergone an axillobifemoral bypass for extensive aortoiliac disease. He was overweight with a rotund abdomen and had previous extensive lower abdominal and right groin surgery in childhood with skin grafting for burns. Emergency magnetic resonance angiography confirmed occlusion of the axillobifemoral bypass and he was taken to theatre urgently for revision reconstruction.

At surgery, the occluded graft was removed with difficulty, retaining the proximal patent stump of the old graft on the axillary artery. Working from the groins, a tunnelling device was passed proximally via a fresh route from the right groin incision to a small subcutaneous relieving incision just above the costal margin and from there to the right infraclavicular region, where a new graft was attached to the tunneller and drawn down to the right femoral incision. Proximally, the new graft was anastomosed to the stump of the old graft and distally, on to the right femoral artery. A crossover limb was fashioned from the main body of the graft to the left femoral artery via a small separate lower abdominal incision. The incisions were closed and the patient was returned to the ward.

Initially, the patient made a good recovery with well perfused legs. However, at 48 hours, he complained of abdominal pain and distension. Bowel sounds were present but an abdominal $x$-ray revealed free gas in the abdomen. The patient was taken back to theatre where a laparotomy revealed that the axillo-right femoral graft component had been inadvertently tunnelled intraperitoneally, directly through the proximal transverse colon, which was adherent to the anterior abdominal wall. The bowel was sealed neatly around the graft with no evident abdominal faecal contamination.

I asked my on-call general surgical colleague to attend briefly to ratify my decision to remove the graft and under- took a temporary defunctioning transverse loop colostomy at the level of the bowel injury. The groins were washed out with copious hydrogen peroxide and saline, and a new axillobifemoral graft was constructed using the contralateral axillary artery as the inflow source. The patient made a satisfactory (albeit protracted) recovery but has not had his colostomy reversed yet.

\section{Reporter's comments}

Neither the registrar nor I were aware of penetration of the abdomen by the rigid metal tunnelling tool, which has a pointed but blunt olive at its tip. Inappropriate force was not employed and, by the level of the costal margin, having skewered the bowel, the tunneller had re-emerged into the subcutaneous plane. Factors contributing to the peritoneal breach and bowel injury included: scarring in the groin from previous burn surgery, which made it difficult to direct the tunneller; the relatively acute angle of the abdominal margin above the groin owing to the patient's habitus; and intra-abdominal adhesions resulting in attachment of the transverse colon to the abdominal wall. Vascular surgeons tunnel grafts regularly and this case highlights the need to maintain vigilance at all times to ensure that inadvertent injury to adjacent structures is not caused by careless use of an invasive instrument.

\section{CORESS comments}

This is a detailed account of an ever-present danger that may occur when traversing tissues blindly with a rigid instrument. Operator awareness of the risks is the key to avoiding this complication. In the presence of a clean perforation of the bowel with no faecal contamination, bowel repair may have sufficed, with placement of a new arterial graft. Although not always feasible, tunnelling from 'north to south' may reduce risk of this type of injury. 


\section{Tunnel vision 2}

A female child was born preterm and suffered with a grade 4 intraventricular haemorrhage. This was monitored clinically and with serial ultrasonography of the head but at six weeks it was noted that head circumference was increasing, and the child was becoming symptomatic with poor feeding and irritability. The child was referred for a neurosurgical opinion. Computed tomography revealed ventriculomegaly and it was decided to place a ventriculoperitoneal shunt to decompress the cerebral ventricles. This was carried out (as per unit practice) by a consultant neurosurgeon at the beginning of the operating list. The procedure was apparently uneventful and the patient returned to the ward after recovering from the anaesthetic. That evening some bogginess was noted around the wound and a simple head bandage was applied to good effect.

Overnight, the patient remained stable but on the ward round the following morning, 'surgical crepitus' was noted on palpation of the neck and scalp. X-rays were undertaken immediately to exclude pneumothorax or free gas in the peritoneum. A small pneumothorax was detected and a chest drain was placed. Free gas was also noted below the diaphragm on an erect abdominal $\mathrm{x}$-ray. The paediatric surgeons were consulted and an exploratory laparotomy was performed, which revealed that the distal ventriculoperitoneal shunt tubing cleanly transfixed the transverse colon. The shunt system was removed and an abdominal drain was placed. The bowel damage was repaired by direct closure of the colonic perforations. The child subsequently developed a ventriculitis, which was managed successfully with intrathecal antibiotics. Finally, a replacement ventriculoperitoneal shunt was placed in situ. The child is now making a good recovery.

\section{CORESS comments}

As in the previous case, care must always be taken when tunnelling through tissue planes blindly. Early recognition of the complication facilitated early correction in this case. A high index of suspicion should be maintained for potential injuries following this type of surgical manoeuvre.

\section{Tunnel vision 3}

A 73-year-old man, who had undergone an aortobifemoral bypass for bilateral iliac artery disease and debilitating claudication 4 weeks previously, presented with blood streaked stools on defaecation. Proctoscopy was normal but sigmoidoscopy revealed a length of dacron graft passing though the lumen of the distal sigmoid. A laparotomy was undertaken (Fig 1). The left limb of the aortobifemoral graft was excised and the sigmoid colon exteriorised temporarily as a loop colostomy. The remainder of the arterial graft appeared well incorporated into surrounding tissues so the proximal stump of the left limb of the graft was oversewn and, after careful groin wound irrigation, a right-toleft femorofemoral cross-over bypass was undertaken. The patient was maintained on long-term antibiotics but made an uneventful recovery.

\section{Reporter's comments}

At surgery, the initial vascular graft was tunnelled from the aorta to the left groin with the aid of a Roberts arterial clip. This must have pierced the sigmoid colon inadvertently and the graft was tunnelled directly through the wall of the colon with a good seal, which prevented faecal leakage and peritonitis. The risks of using forcible rigid devices blind- ly as an aid to tunnelling a passage for a conduit are selfevident. The patient was lucky to have avoided overt septic complications and peritonitis although in the long term, graft infection remains a risk.

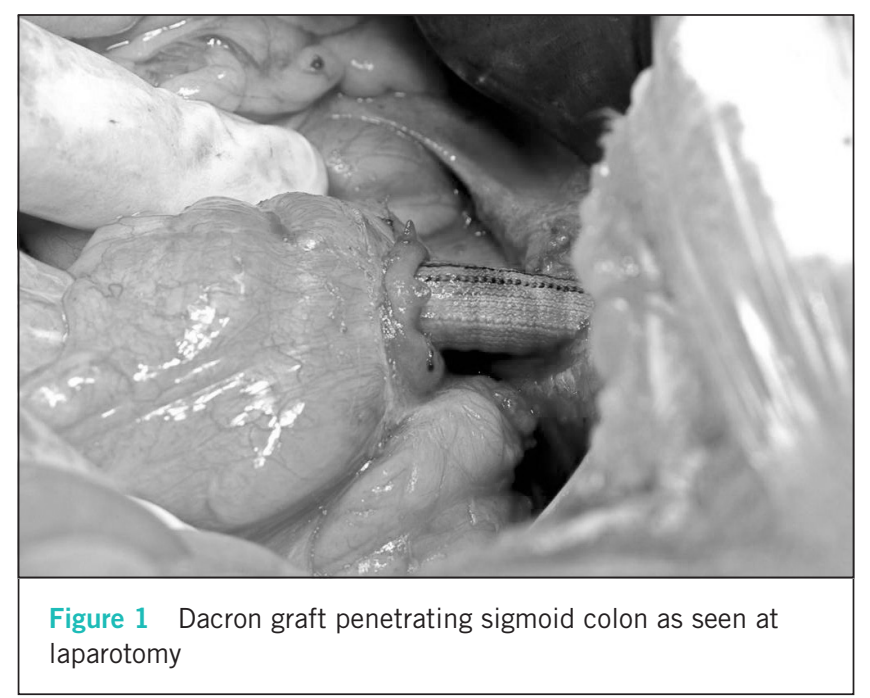




\section{Tunnel vision 4}

A 75-year-old man with debilitating left thigh, buttock and calf claudication underwent a right-to-left femorofemoral bypass for a long left iliac artery occlusion that was not amenable to angioplasty. The graft was tunnelled over the pubis using a long arterial clip. Postoperatively, the patient made a satisfactory initial recovery but was noted to have developed frank haematuria, which was evident in the urine collection bag on the morning after surgery. The urinary catheter was removed and early cystoscopy revealed a dacron graft
(Ref 148)

passing through the vault of the bladder. The graft had been tunnelled inadvertently through the bladder while trying to avoid scar tissue from a previous midline laparotomy.

\section{CORESS comments}

The lessons learned from the previous cases apply equally to this case. 